\title{
Information processing for remote recognition of the state of bee colonies and apiaries in precision beekeeping (apiculture)
}

\author{
Aleksejs Zacepins ${ }^{1,2 *}$, Egils Stalidzans ${ }^{2,3}$ \\ ${ }^{1}$ Computer Control Group, Department of Computer Systems, Latvia University of Agriculture, Liela iela 2, LV-3001, Jelgava, \\ Latvia \\ ${ }^{2}$ SIA TIBIT, Dobeles iela 10-9, LV-3001, Jelgava, Latvia \\ ${ }^{3}$ Biosystems Group, Department of Computer Systems, Latvia University of Agriculture, Liela iela 2, LV-3001, Jelgava, Latvia \\ *Corresponding author \\ alzpostbox@gmail.com
}

Received: 25 May 2013; accepted: 30 May 2013; published online: 30 May 2013.

This paper has no supplementary material.

\begin{abstract}
The principles of Precision Agriculture (PA) can be applied also to the beekeeping branch. Precision Beekeeping (PB) (Precision Apiculture) can be implemented as a three phase cycle including 1) data collection, 2) data analysis and 3) application. The first two phases are based information technologies in case of remote recognition. The third phase is realized manually according to the decisions made after data collection and analysis.

This study is dedicated to the information processing approaches taking into account the peculiarities of the beekeeping branch. Classification of deviations at several levels is proposed: colony level (most colonies in the same location behave normally); apiary level (most apiaries at other locations behave normally); bee farm level (most apiaries of other bee farms behave normally) and regional level (most bee farms in the region do not behave normally). Two levels of information analysis are suggested: bee farm level (information about individual colonies in different apiaries) and regional level (summary of information collected at the level of bee farms).

Decision support systems (DSS) are proposed to automate data analysis. Continuous operation and high processing capacities of electronics can significantly improve implementations of PB. DSS may be delegated to make some decisions automatically or request the analysis of proposed decision by a specialist in data processing or beekeeper.
\end{abstract}

Keywords: precision beekeeping, precision apiculture, data collection, decision support system.

\section{Introduction}

Information and communication technologies (ICT) provide indispensable support for business, agriculture, and production processes. The rapid development of information technologies and computer control enabled the development of precision agriculture (PA) aiming to monitor and control individual agricultural units. The definition of Precision Agriculture is still developing and improving, because technologies that are used in PA are changing and comprehension about theoretical and practical opportunities is developing. Over the years the emphasis of the definition has changed from following soil characteristics in agriculture (Robert and Stafford, 1999) to more complicated where quality of the end product and impact on the environment becomes more relevant (McBratney et al., 2005).

PA principles have been adapted to several agricultural (McBratney et al., 2005; Morais et al., 2008; Whelan and McBratney, 2000) and forestry (Zhang et al., 2011) branches. The same principles can be applied also for beekeeping taking a bee colony as the smallest industrial unit of interest in beekeeping. Apiculture (Beekeeping) is one of the branches of agriculture where precision approach is recently adapted (Zacepins et al., 2012). Precision beekeeping (PB) approach is based on the continuous measurements of individual bee colo- nies and can be applied all year round thus detecting different states of colonies and apiaries enabling rapid reaction by the beekeeper in case of necessity (Zacepins et al., 2012).

PA branches can be analyzed as a three phase cycle including 1) data collection, 2) data analysis and 3) application (Terry, 2006). These phases are at very different development stage in case of PB. Thre first two phases are closely related to the information technologies while the third one usually has to be done by a beekeeper according to the decisions made after data anlysis.

There are quite many parameters that can be measured to assess the state of individual bee colonies. Still they are very different in terms of information processing and transmission. For instance a temperature measurement returns just one number that can be easily stored in memory or transmitted while sound measurements request intensive processing or transmission of large amount of data.

The data analysis phase is the stumbling block to adoption of PA generally (McBratney et al., 2005). The same applies to the precision beekeeping. Some data analysis based decision support systems are reported in the literature. Most of them concentrate on single colony level while the others are aiming for benefit of larger regions involving wider community into 
measurements and data exchange enabled by information technology.

The article concentrates on the remote performance of measurements, data analysis of measurements and principles of decision support systems taking into account the peculiarities of beekeeping branch and opportunities offered by information technologies.

\section{Different level data based state recognition in precision beekeeping}

Compared to other branches of agriculture the industrial beekeeping has several peculiarities which should be addressed by PB technologies. (1) Honey bees are social insect and one industrial unit is a bee colony that consists of tens of thousands of individual bees. (2) The foraging area of honey bees is around their location within radius of about 3 kilometres and beekeeper can influence the feedstock by transporting bee colonies to places with different nectar sources. (3) Bee colonies usually are kept in groups with limited number of 1030 colonies in one location because more colonies may not have sufficient amount of nectar available in the foraging area which leads to reduced incomes per colony. (4) Remote state recognition is important because bee colonies in apiaries can be left without inspection for long time if they are in acceptable state. (5) Wide foraging area adds complexity to the control of bee diseases.

A beekeeper is interested in classification of deviations at several levels taking into account the above mentioned peculiarities and business tasks of beekeeping (Fig. 1): colony level (most colonies in the same location behave normally); apiary level (most apiaries at other locations behave normally); beekeeper's farm level (most apiaries of other bee farms behave normally) and regional level (most bee farms in the region do not behave normally).

To operate with data at different levels it is necessary to centralize the data by it's transfer using internet or other transmission technologies depending on the local circumstances to extract maximal benefit from any measurement. The value of a single measurement may increase analyzing it in context with other ones.
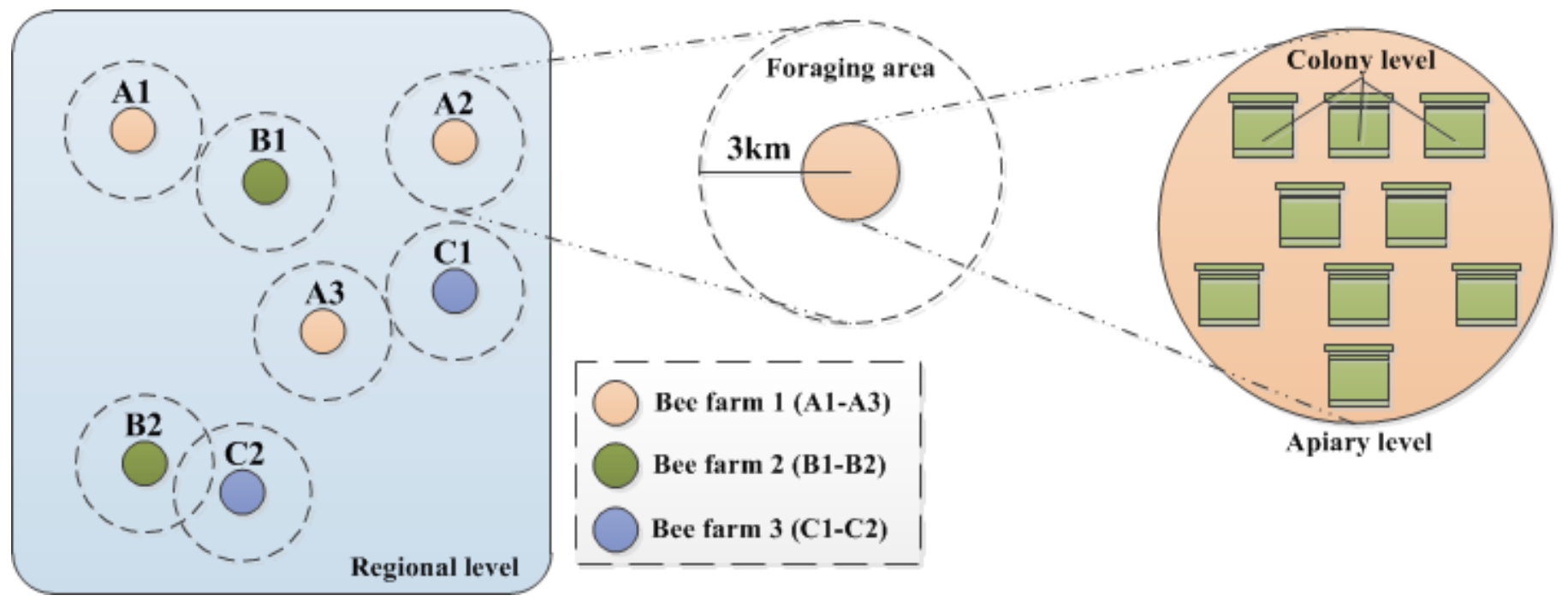

Fig. 1. Different scales of information collection in beekeeping: colony level, apiary level, bee farm level and regional level.

\subsection{Colony level decisions}

Colony level decisions should be made based on individual colony measurements and monitoring. For example, based on low temperature in a colony it is possible to conclude if the bee colony is in a passive/inactive state, if other colonies have temperature about $30^{\circ} \mathrm{C}$ (Stalidzans and Berzonis, 2013). Preswarming and swarming state detection is another colonylevel challenge for automatic remote detection systems.

Temperature measurements of the individual colonies seem to be the most cost efficient way to monitor colony activity and behaviour (Zacepins and Karasha, 2013; Zacepins et al., 2013). Other parameters like air humidity, gas content, sound, video and may be used as well. Still analysis of economical feasibility of different systems has to be clarified depending on technological, climatic and genetic context of particular bee farms or even apiaries.

\subsection{Apiary level decisions}

Apiary level problems are mainly related to the location of apiary assuming that all the apiaries of particular bee farm are treated in the same way. In this case all the apiary colonies are exposed to the apiary specific factor. Some examples of apiary level factors are: limitations of nectar availability, application of pesticides within the foraging area, noise or other disturbances close to the apiary, theft, diseases.

In spite of the fact that all the colonies in apiary are exposed to the disturbing factor their reaction may be different depending on the internal state of colonies (after swarming, queenless etc.). Video technologies can be used for apiary level monitoring to observe the whole apiary. Different approaches of video activation can be used to reduce the amount of produced data (Meitalovs et al., 2009) if necessary. Climate observation tools with remote connectin can be applied to determine the local weather parameters.

According to the measurements apiary level decisions can be very different: visit of the apiary to examine the situation in details, transportation of bee colonies to a different place, feeding of bee colonies, disease treatment etc.

\subsection{Bee farm level decisions}

Farm specific problems mostly are caused by the way of operation of bee farm and should be observed in all the 
apiaries belonging to the same bee farm (Fig. 1). The causes should be of technological origin: wrong timing of operations, inefficient medical treatment etc.

The decisions should be based on analysis of the applied technologies and approaches if similar deviations are not observed at apiaries of a different bee farms having apiaries in the same area which should be exposed to similar circumstances (for instance B2 and C2 in Fig.1). Thus it is critical for farm level decisions to have access to the measurements of other farms in the same region to distinguish between bee farm and regional level problems as they may require different decisions and actions.

One example of remote decision system is practically applied for indoor wintering of bees where temperature monitoring at individual colony level is proposed (Zacepins and Stalidzans, 2012). This kind of architecture can be used both for apiary and farm level (Fig. 2).

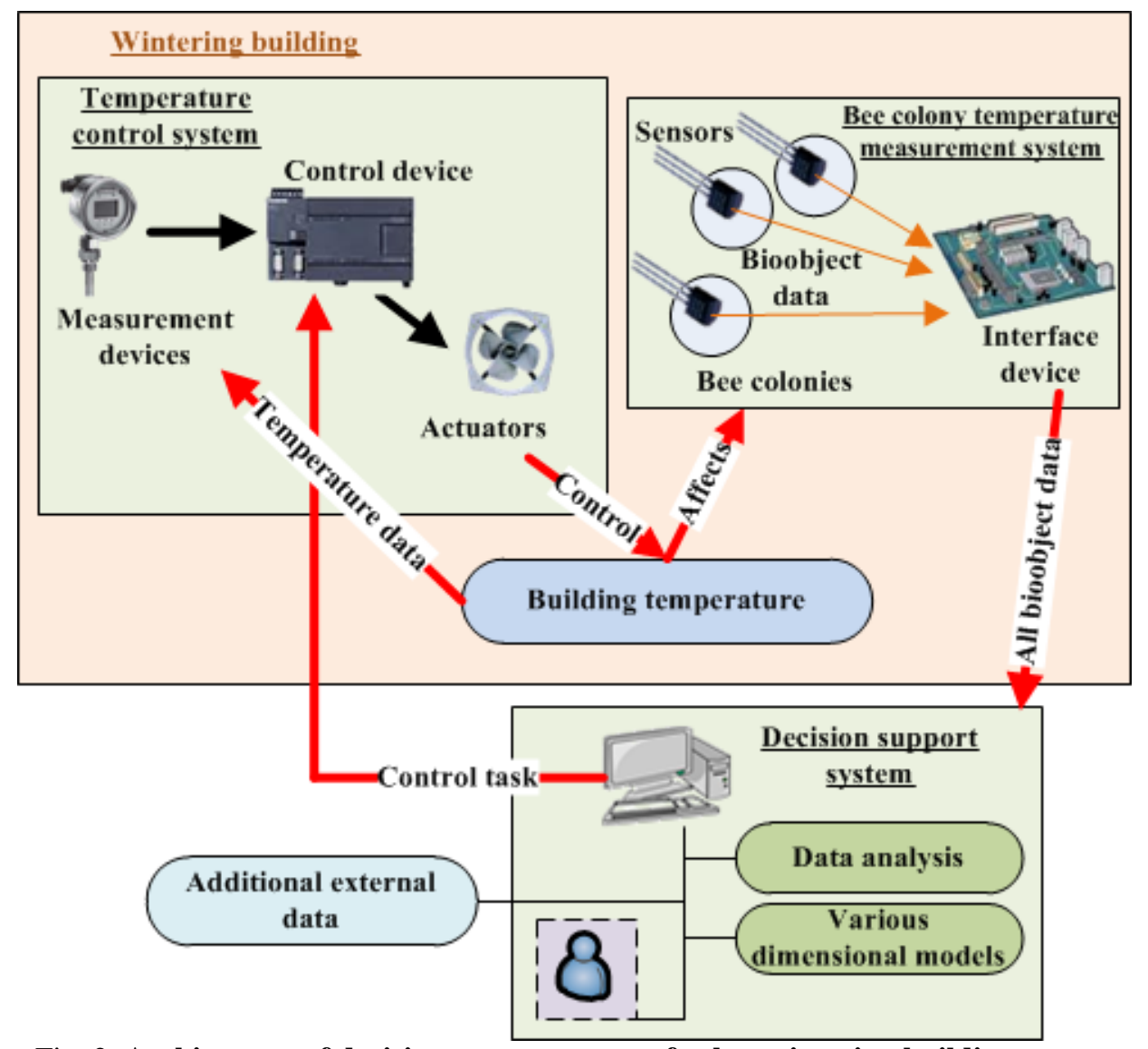

Fig. 2. Architecture of decision support system for bee wintering building.

\subsection{Regional level based decisions}

Similar deviations from normal behaviour of significant part of apiaries of different farms in the same geographical region are a signal for necessary measures at regional scale. Regional level problems can be caused by unusual climatic circumstances (dry, wet, cold, hot), diseases of bees or plants etc. Information about regional problems, detected by collaboration of several bee farms by the exchange of measurements, can be spread among all the beekeepers (including hobby ones) in the region even if they do not participate in the collection of measurements. Informing about regional problems some activities can be suggested to minimize the impact of discovered regional problems.

Early diagnostics of diseases, especially infectious ones can prevent significant losses in a region directly for beekeepers and indirectly for agriculturists due to reduced pollination. Colony collapse disorder (CCD) may serve as a good example for regional level problems (Cox-Foster et al., 2007; Van Engelsdorp et al., 2008).

Example of functioning regional level data collection system prototype is transnational bee colony monitoring system which operates as an international network including bee colonies in Denmark, Sweden, Norway, Latvia and Germany (http://biavl.volatus.de/bsm0/BSM.html\#). The advantage of this system is it's geographical coverage and the number of measured parameters: weight changes, ambient temperature, precipitation, and the temperature of the colony. A disadvantage is the low number of monitored colonies and lack of decision support system. Independent on it's current execution and intensity of use this system is a good prototype for future developments.

Another regional level project is NASA Goddard Space Flight Center initiated project (http://honeybeenet.gsfc.nasa.gov/) where daily weighing of hives by volunteers is merged with satellite data (Nightingale et al., 2008). Beekeepers can also directly monitor the weight changes to estimate the amount of incoming nectar.

Mentioned projects indicate both interest in remote data collection and sharing and technical opportunities for practical implementation at a regional level. Still reliable decision support systems are needed to make use of collected data. 


\section{ICT aspects of information collection and decision support in PB}

\subsection{Information collection, processing and transfer}

A prerequisite of the above mentioned four level based decisions is a system of information collection, processing and transfer. Two levels of information collection can be used: bee farm level (information about individual colonies in different apiaries) and regional level (summary of information at the level of bee farms). Bee farm level information collection is reasonable as the lowest level of decision making assuming that all the apiaries of particular bee farm is managed by the same team. The collection and analysis of regional information can be performed by regional governments, beekeeper societies or temporary projects.

While developing farm level systems it is crucial to decide about information processing and transfer options, because it is possible to process data onsite and transfer just a summary or transfer all the raw data to a remote computational centre for further processing. The decision about local or centralized information processing can significantly influence the costs of system. That can be very important issue depending on the processing peculiarities of particular parameter. For instance, the result of temperature or humidity measurements is just a digit that does not request much processing and even transfer of that information is cheap. That is different in case of sound measurements or video recording where both processing and transfer are much more complicated and costly.

Apiaries which are located in sites without centralized electricity supply have another problem: energy source. Depending on a solution (batteries, solar panel, wind generator etc.) additional limitations may appear having impact on the feasibility of PB system of interest (Zacepins et al., 2013).

\subsection{Decision support systems (DSS)}

The second stage of PA approach - data analysis - can be performed by the beekeeper interpreting received data. Information technologies can at least partly replace a specialist in case of large data amount or if continuous analysis is necessary. such approach could help beekeepers which hardly could interpret the data by themselves. The computational support of beekeepers can be done using DSS where different algorithms and models can be implemented (Fig. 3).

Task of the models is to represent various real physical, biological, economical or other processes. Usually models give a simplified view about process, but nevertheless information, which is provided by the model, is useful for the detailed research of the process (Sokolowski and Banks, 2009). Models can be divided in two categories: identification (qualitative) and quantitative models (Holjushkin and Grazhdannikov, 2000). For instance, identification model could be used to determine if colony is in the preswarming state where the answer is "yes" or "no". A quantitative model would predict, for instance, the number of bees in the colony in particular date where the answer is a number of bees.

DSS may use different combinations of different model types to suggest particular decisions to the beekeeper.

Authors propose to divide decision making process in three levels (Fig. 4):

- input data level - where all needed data about process and object should be defined;

- model level - where input data is used by various different dimension models with main aim to determine the object state and status of the process;

- decision level - where model outputs are analysed with main aim to choose the right decision (beekeeping operation to be performed).

Different states of colony, apiary, bee farm and region can be recognized with different level of reliability. Therefore, depending on the importance of detected state and importance of immediate action DSS may be delegated to make some decisions automatically or request the analysis of proposed decision by a specialist in data processing or beekeeper. Thus the combination of ICT applications in data collection and data analysis can give important new tools for PB applications at bee farm and regional level.

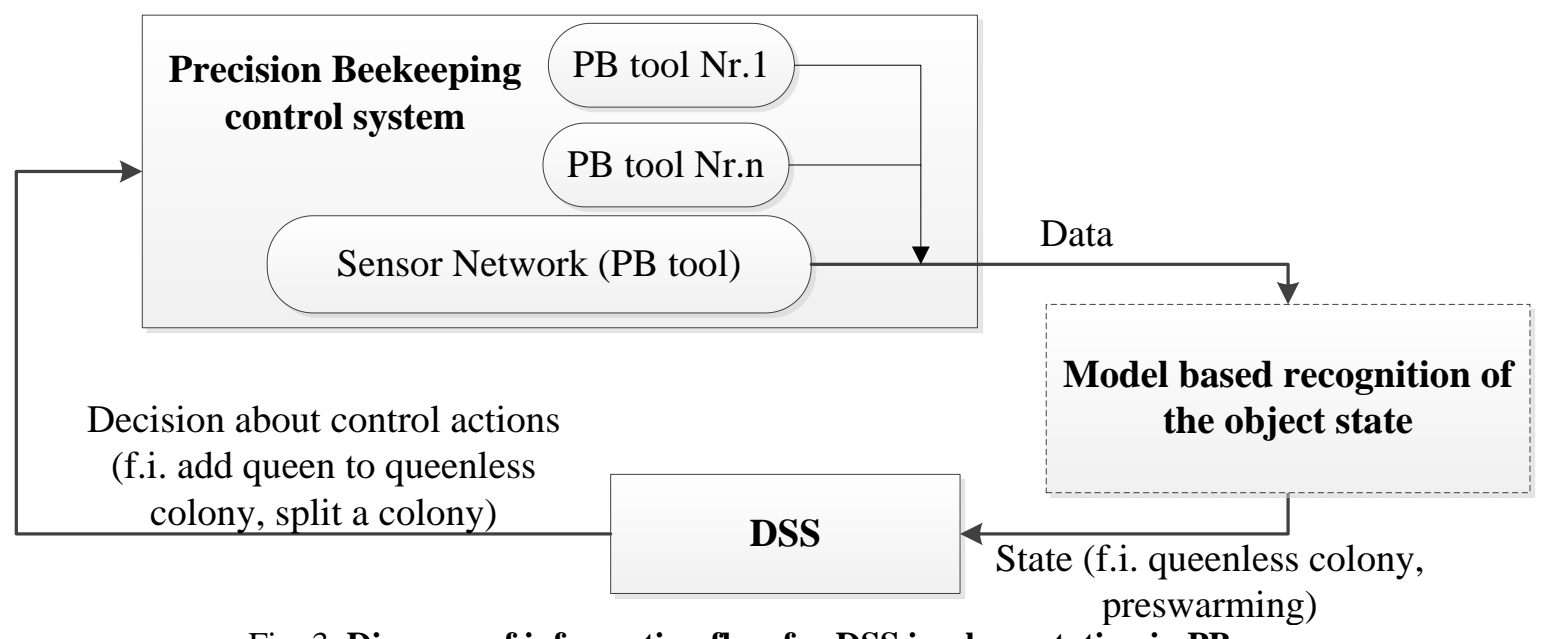

Fig. 3. Diagram of information flow for DSS implementation in PB. 


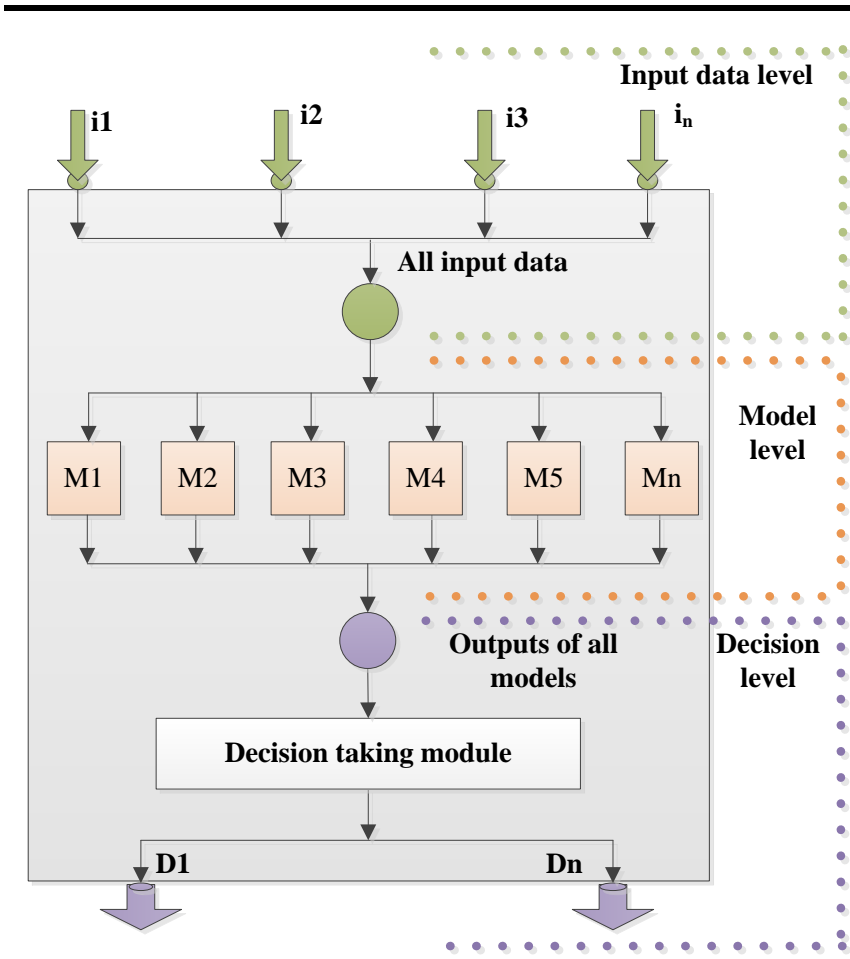

Fig. 4. Three levels of the decision making in DSS.

\section{Conclusion}

ICT can be applied at two phases of Precision Beekeeping (PB): data collection and data analysis. Data collection includes the data collection on the colony level, data processing and data transfer to make them available for the specialist or decision support system. Rational compromise between local processing of information and transmission of unprocessed information has to be found depending on several factors.

Decision support systems (DSS) are proposed to automate data analysis. Continuous operation and high processing capacities of electronics thus can be useful help in PB. DSS may be delegated to make some decisions automatically or request the analysis of proposed decision by a specialist in data processing or beekeeper.

Several levels of decisions can be made using PB approach based on different level of information: colony level, apiary level, bee farm level and regional level. Two levels of information analysis can be used: bee farm level (information about individual colonies in different apiaries) and regional level (summary of information collected at the level of bee farms). Bee farm level information collection is reasonable as the lowest level of decision assuming that all the apiaries of particular bee farm is managed by the same team. The collection and analysis of regional information can be performed by regional governments, beekeeper societies or temporary projects.

Three level decision making process is proposed: input data level, model level and decision level. It is proposed to use interaction between quantitative and qualitative models.

\section{References}

Cox-Foster, D.L., Conlan, S., Holmes, E.C., Palacios, G., Evans, J.D., Moran, N. a, Quan, P.-L., et al. (2007), "A metagenomic survey of microbes in honey bee colony collapse disorder.," Science (New York, N.Y.), Vol. 318 No. 5848, pp. 283-7. http://dx.doi.org/10.1126/science.1146498

Van Engelsdorp, D., Hayes, J., Underwood, R.M. and Pettis, J. (2008), "A survey of honey bee colony losses in the U.S., fall 2007 to spring 2008.,"
PloS one, Vol. 3 No. 12, p. e4071. http://dx.doi.org/10.1371/journal.pone.0004071

Holjushkin, J.P. and Grazhdannikov, E.D. (2000), "Sistemnaja klassifikacija arheologicheskoj nauki," Klassifikacionnue modeli v arheologicheskom naukovedenie.

McBratney, A., Whelan, B., Ancev, T. and Bouma, J. (2005), "Future directions of precision agriculture," Precision Agriculture, Vol. 6, pp. $7-$ 23.

Meitalovs, J., Histjajevs, A. and Stalidzans, E. (2009), "Automatic Microclimate Controlled Beehive Observation System," 8th International Scientific Confernce "Enginieering for Rural Development", Jelgava, Latvia, Latvia University of Agriculture, pp. 265-271.

Morais, R., Fernandes, M. a., Matos, S.G., Serôdio, C., Ferreira, P.J.S.G. and Reis, M.J.C.S. (2008), "A ZigBee multi-powered wireless acquisition device for remote sensing applications in precision viticulture,' Computers and Electronics in Agriculture, Vol. 62 No. 2, pp. 94-106. http://dx.doi.org/10.1016/j.compag.2007.12.004

Nightingale, J.M., Esaias, W.E., Wolfe, R.E., Nickeson, J.E. and Ma, P.L.A (2008), "Assessing Honey Bee Equilibrium Range and Forage Supply using Satelite-Derived Phenology," IGARSS 2008 - 2008 IEEE International Geoscience and Remote Sensing Symposium, IEEE, pp. III 763-III - 766. http://dx.doi.org/10.1109/IGARSS.2008.4779460

Robert, P. and Stafford, J. (1999), "Precision Agriculture: research needs and status in the USA.," Conference on Precision Agriculture, pp. 19-33.

Sokolowski, J.A. and Banks, C.M. (2009), Principles of Modeling and Simulation, New York, Wiley, p. 257. http://dx.doi.org/10.1002/9780470403563.index

Stalidzans, E. and Berzonis, A. (2013), "Temperature changes above the upper hive body reveal the annual development periods of honey bee colonies,' Computers and Electronics in Agriculture, Vol. 90, pp. 1-6. http://dx.doi.org/10.1016/j.compag.2012.10.003

Terry, B. (2006), Precision Agriculture, Thomson Delmar learning, p. 224

Whelan, B.M and McBratney, A.B. (2000), "The 'null hypothesis' of precision agriculture management," Precision Agriculture, Vol. 2, pp. 265-279.

Zacepins, A. and Karasha, T. (2013), "Application of temperature measurements for the bee colony monitoring: a review," Proceedings of the 12th International Scientific Conference "Engineering for Rural Development", Jelgava, Latvia, pp. 126-131.

Zacepins, A., Stalidzans, E. and Karasha, T. (2013), "Profitability ranking of precision agriculture measurement systems implementation," Proceedings of the 12th International Scientific Conference "Engineering for Rural Development", Jelgava, Latvia, pp. 164-169.

Zacepins, A. and Stalidzans, E. (2012), "Architecture of automatized control system for honey bee indoor wintering process monitoring and control," Proceedings of 13th International Carpathian Control Conference (ICCC), 28-31 May 2012, High Tatras, pp. 772-775.

Zacepins, A., Stalidzans, E. and Meitalovs, J. (2012), "Application of Information Technologies in Precision Apiculture," Proceedings of the 11th International Conference on Precision Agriculture, 15-18 July 2012, Indianapolis, IN, USA.

Zhang, Q., Li, J. and Rong, J. (2011), “Application of WSN in precision forestry," IEEE 2011 10th International Conference on Electronic Measurement \& Instruments, IEEE, pp. 320-323. http://dx.doi.org/10.1109/ICEMI.2011.6038006 